\title{
Open Bose-Hubbard chain: Pseudoclassical approach
}

\author{
A. A. Bychek, ${ }^{1}$ P. S. Muraev $\odot,{ }^{2}$ D. N. Maksimov $\odot,{ }^{1,2}$ and A. R. Kolovsky $\odot^{1,2}$ \\ ${ }^{1}$ Kirensky Institute of Physics, 660036 Krasnoyarsk, Russia \\ ${ }^{2}$ School of Engineering Physics and Radio Electronics, Siberian Federal University, 660041 Krasnoyarsk, Russia
}

(Received 17 October 2019; published 13 January 2020)

\begin{abstract}
We analyze the stationary current of bosonic carriers in the Bose-Hubbard chain of length $L$ where the first and the last sites of the chain are attached to reservoirs of Bose particles acting as a particle source and sink, respectively. The analysis is carried out by using the pseudoclassical approach which reduces the original quantum problem to the classical problem for $L$ coupled nonlinear oscillators. It is shown that an increase of oscillator nonlinearity (which is determined by the strength of interparticle interactions) results in a transition from the ballistic transport regime, where the stationary current is independent of the chain length, to the diffusive regime, where the current is inversely proportional to $L$.
\end{abstract}

DOI: 10.1103/PhysRevE.101.012208

\section{INTRODUCTION}

Transport phenomena with neutral atoms is an active area of research in experimental cold-atom physics. These studies include Josephson oscillations of Bose atoms in a doublewell potential [1,2], atomic Bloch oscillations in quasi-onedimensional optical lattices [3,4], refill dynamics in the presence of induced losses [5,6], and quantized current in the engineered transport channel [7-9], to mention a few of the phenomena under scrutiny. From the theoretical viewpoint these experiments refer to (or even are aimed to realize) some paradigm models of the many-body physics that, on the one hand, are complex enough to capture the physics of the discussed phenomenon but, on the other hand, are simple enough to admit an analytical treatment. The present work analyzes one of these paradigm models, namely, the open or dissipative Bose-Hubbard (BH) chain [10-13]. With respect to cold atoms this model describes the atomic current between two reservoirs of Bose atoms which are connected by a deep optical lattice. More formally, the model assumes that the system dynamics is governed by the master equation for the reduced density matrix $\mathcal{R}(t)$ of the bosonic carriers,

$$
\frac{d \mathcal{R}}{d t}=-i[\widehat{H}, \mathcal{R}]+\mathcal{L}_{1}(\mathcal{R})+\mathcal{L}_{L}(\mathcal{R}),
$$

where $\widehat{H}$ is the Hamiltonian of the closed (isolated) BH model,

$$
\widehat{H}=\omega \sum_{l=1}^{L} \hat{n}_{l}-\frac{J}{2} \sum_{l=1}^{L-1}\left(\hat{a}_{l+1}^{\dagger} \hat{a}_{l}+\text { H.c. }\right)+\frac{U}{2} \sum_{l=1}^{L} \hat{n}_{l}\left(\hat{n}_{l}-1\right),
$$

and the relaxation operator $\mathcal{L}_{1}(\mathcal{R})$ acting on the first site of the chain and the operator $\mathcal{L}_{L}(\mathcal{R})$ acting on the last site of the chain have the standard Lindblad form parametrized by the relaxation constant $\gamma_{1}\left(\gamma_{L}\right)$ and the particle density $\bar{n}_{1}$ $\left(\bar{n}_{L}\right)$ of the respective reservoir [see Eqs. (5) and (6) below]. To be certain we assume $\bar{n}_{1}>\bar{n}_{L}$; then the left reservoir acts as a particle source and the right reservoir as a particle sink. The goal is to find the stationary current $\tilde{j}$ of bosonic particles in the chain as a function of the reservoir parameters and parameters of the $\mathrm{BH}$ chain. We also mention similar problems for the stationary current of the fermionic carriers and heat transport in the spin chain [14-17].

Without additional assumptions or approximations the formulated problem can be solved only for vanishing interparticle interactions $(U=0)$, where one obtains a relatively simple analytical expression for the stationary current $[10,13]$. As expected, it appears to be proportional to the difference in the reservoir densities, $\bar{n}_{1}-\bar{n}_{L}$, and is independent of the chain length $L$. The case of finite interactions, $U \neq 0$, however, remains a challenge. In particular, it is not clear whether the system is conducting or becomes insulating in the limit $L \rightarrow \infty$. In this work we analyze this problem by using the so-called pseudoclassical approach [18-25], which is justified for large occupation numbers of the chain sites and was proved to be successful in application to a number of other problems, such as dynamics of Bose-Einstein condensates in the double-well potential $[23,25]$ and Bloch oscillations of interacting Bose atoms [22]. The advantage of the pseudoclassical approach is that it provides a clear insight into the physics of the discussed phenomenon by mapping the quantum many-body system into a classical (generally multidimensional) system. Using this approach we show that an increase of the interaction constant from zero to a finite value $U \sim J / \bar{n}_{1}$ results in a transition from the ballistic transport regime, where the stationary current $\tilde{j}$ is independent of the chain length $L$, to the diffusive regime, where $\tilde{j} \sim 1 / L$.

\section{PSEUDOCLASSICAL APPROACH}

To illustrate the main ideas of the pseudoclassical approach we revisit the problem of the damped nonlinear oscillator,

$$
\widehat{H}=\omega \hat{a}^{\dagger} \hat{a}+\frac{U}{2}\left(\hat{a}^{\dagger} \hat{a}\right)^{2},
$$

where we set the fundamental Planck constant to unity, i.e., $\left[\hat{a}, \hat{a}^{\dagger}\right]=1$. 


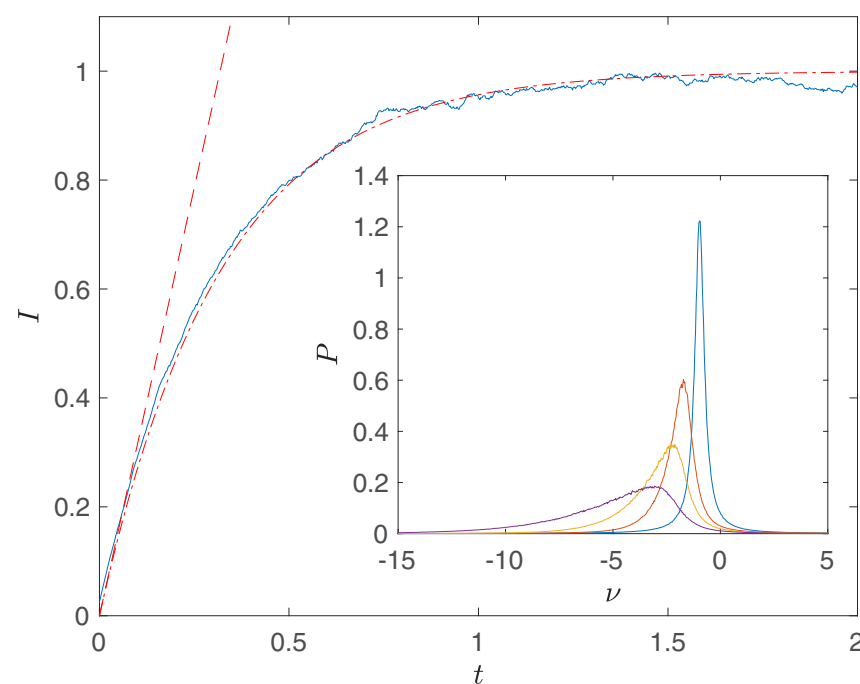

FIG. 1. Dash-dotted line: Dynamics of $I(t)=N(t) / \bar{n}$ according to Eq. (4). Parameters are $\gamma=0.5$ and $\bar{n}=10$. The initial condition corresponds to the oscillator ground state, i.e., to the Fock state $|n\rangle$ with $n=0$. Dashed line: Solution of master equation (7) for $D=0.5$ and $\gamma=0$. Solid blue line: Solution of the Langevin equation (20) averaged over 4000 realizations of the random process $\xi(t)$. The inset shows the oscillator spectral density $P(v)$ for $\omega=1$ and different values of nonlinearity $g=0,0.5,1,2$, from top to bottom.

\section{A. Quantum analysis}

Commonly the dissipative dynamics of system (3) is described by the following master equation:

$$
\frac{d \mathcal{R}}{d t}=-i[\widehat{H}, \mathcal{R}]+\mathcal{L}_{\text {gain }}(\mathcal{R})+\mathcal{L}_{\text {loss }}(\mathcal{R}),
$$

where the Lindblad operators $\mathcal{L}_{\text {loss }}$,

$$
\mathcal{L}_{\text {loss }}(\mathcal{R})=-\frac{\gamma(\bar{n}+1)}{2}\left(\hat{a}^{\dagger} \hat{a} \mathcal{R}-2 \hat{a} \mathcal{R} \hat{a}^{\dagger}+\mathcal{R} \hat{a}^{\dagger} \hat{a}\right),
$$

and $\mathcal{L}_{\text {gain }}$,

$$
\mathcal{L}_{\text {gain }}(\mathcal{R})=-\frac{\gamma \bar{n}}{2}\left(\hat{a} \hat{a}^{\dagger} \mathcal{R}-2 \hat{a}^{\dagger} \mathcal{R} \hat{a}+\mathcal{R} \hat{a} \hat{a}^{\dagger}\right),
$$

take into account the exchange of particles between the system and bosonic reservoir characterized by the parameter $\bar{n}$. It is easy to prove that the Lindblad operators (5) and (6) ensure relaxation of the oscillator to the steady state with the mean occupation number given by the parameter $\bar{n}$. As an example, the dash-dotted red line in Fig. 1 shows the solution of Eq. (4) for $\bar{n}=10$ and $\gamma=0.5$ where we choose the oscillator ground state as the initial condition. For this specific initial condition relaxation to the steady state has a particularly simple time dependence $N(t)=\operatorname{Tr}\left[\hat{a}^{\dagger} \hat{a} \mathcal{R}(t)\right]=$ $\bar{n}[1-\exp (-\gamma t)]$.

To get a deeper insight into the discussed relaxation process it is instructive to rewrite Eq. (4) in the following form:

$$
\frac{d \mathcal{R}}{d t}=-i[\widehat{H}, \mathcal{R}]+\mathcal{D}(\mathcal{R})+\mathcal{G}(\mathcal{R}),
$$

where

$$
\mathcal{D}(\mathcal{R})=-\frac{D \bar{n}}{2}\left(\left[\hat{a},\left[\hat{a}^{\dagger}, \mathcal{R}\right]\right]+\left[\hat{a}^{\dagger},[\hat{a}, \mathcal{R}]\right]\right)
$$

and

$$
\mathcal{G}(\mathcal{R})=-\frac{\gamma}{2}\left(\hat{a} \hat{a}^{\dagger} \mathcal{R}-2 \hat{a}^{\dagger} \mathcal{R} \hat{a}+\mathcal{R} \hat{a} \hat{a}^{\dagger}\right)
$$

Here, as it will be clear in a moment, the operator (8) is the diffusion term with the diffusion coefficient $D=\gamma$ and the operator (9) is the friction term with the friction coefficient $\gamma$. We mention that in present-day laboratory experiments with cold Bose atoms the diffusion and friction coefficients can be varied independently. Then, for $\gamma=0$ we have pure diffusion (see dashed line in Fig. 1) while for $D=0$ we have pure decay. In the general case an interplay between these processes leads to the steady state where

$$
\lim _{t \rightarrow \infty} N(t)=\bar{n} \frac{D}{\gamma} .
$$

\section{B. Classical analysis}

Let us reproduce the result in Eq. (10) by using the pseudoclassical approach. In this approach the operators $\hat{a}^{\prime}=\hat{a} / \sqrt{\bar{n}}$ and $\hat{a}^{\prime \dagger}=\hat{a}^{\dagger} / \sqrt{\bar{n}}$, which commute to the effective Planck constant $\hbar^{\prime}=1 / \bar{n}$,

$$
\left[\hat{a}^{\prime}, \hat{a}^{\prime \dagger}\right]=\hbar^{\prime}, \quad \hbar^{\prime}=1 / \bar{n} \ll 1,
$$

are identified with the pair of canonical variables $a$ and $a^{*}$ :

$$
a=(q+i p) / \sqrt{2}, \quad a^{*}=(q-i p) / \sqrt{2} .
$$

Applying the Wigner-Weyl transformation to master equation (7), and keeping only the zero-order terms in the expansion over the effective Planck constant $\hbar^{\prime}$, we obtain the following equation for the Wigner function $f=f\left(a, a^{*} ; t\right)[26,27]$ :

$$
\frac{\partial f}{\partial t}=\{H, f\}+\mathcal{D}(f)+\mathcal{G}(f),
$$

where the Hamiltonian evolution is captured by the Poisson brackets with the Hamiltonian

$$
H=\omega a^{*} a+\frac{g}{2}\left(a^{*} a\right)^{2}, \quad g=U \bar{n},
$$

the diffusion term is

$$
\mathcal{D}(f)=D \frac{\partial^{2} f}{\partial a \partial a^{*}},
$$

and the friction term is given by

$$
\mathcal{G}(f)=\frac{\gamma}{2}\left(a \frac{\partial f}{\partial a}+2 f+a^{*} \frac{\partial f}{\partial a^{*}}\right) .
$$

The obtained Fokker-Planck equation [Eq. (13)] has a simple analytical solution if the initial distribution function is given by the two-dimensional Gaussian

$$
\begin{aligned}
f\left(a, a^{*} ; t=0\right) & =\frac{1}{2 \pi i \sigma^{2}} \exp \left(-\frac{|a|^{2}}{\sigma^{2}}\right) \\
& =\frac{1}{2 \pi \sigma^{2}} \exp \left(-\frac{q^{2}+p^{2}}{2 \sigma^{2}}\right) .
\end{aligned}
$$


Substituting this ansatz with $\sigma^{2}=\sigma^{2}(t)$ in Eq. (13) we find that $\sigma^{2}(t)$ grows linearly if $\gamma=0$ or decays exponentially if $D=0$. Correspondingly, the mean oscillator action $I(t)$,

$$
I(t)=\iint|a|^{2} f\left(a, a^{*} ; t\right) d a d a^{*}
$$

grows as $I(t)=I_{0}+D t$ if $\gamma=0$ or decays as $I(t)=$ $I_{0} \exp (-\gamma t)$ if $D=0$. In the general case the oscillator approaches the steady state where

$$
\lim _{t \rightarrow \infty} I(t) \equiv \tilde{I}=\frac{D}{\gamma}
$$

Notice that Eq. (19) holds for arbitrary oscillator nonlinearity $g$.

\section{Stochastic method}

For the purpose of future reference we discuss the Monte Carlo method of solving Eq. (13). It is easy to prove that the Fokker-Planck equation [Eq. (13)] is equivalent to the following Langevin equation:

$$
i \dot{a}=\frac{\partial H}{\partial a^{*}}-i \frac{\gamma}{2} a+\sqrt{\frac{D}{2}} \xi(t),
$$

where $\xi(t)=\operatorname{Re}[\xi(t)]+i \operatorname{Im}[\xi(t)]$ is the $\delta$-correlated noise with standard deviation equal to unity for both the real and imaginary parts. Then the mean value of action in Eq. (18) can be calculated as the average over different realizations of the random process $\xi(t)$, namely, $I(t)=\overline{|a(t)|^{2}}$. As an example, the solid blue line in Fig. 1 shows the mean action which is obtained by using 4000 realizations of the random process. Increasing the number of realizations by one order of magnitude makes the result practically indistinguishable from the exact solution.

Importantly, the described stochastic approach allows us to introduce the other important characteristic of the systemthe oscillator spectral density $P(v)[28,29]$,

$$
P(v)=\overline{|a(v)|^{2}}, \quad a(v)=\lim _{T \rightarrow \infty} \frac{1}{T} \int_{0}^{T} a(t) e^{i v t} d t .
$$

Here the limit $T \rightarrow \infty$ ensures that $P(v)$ refers to the stationary regime. In practice this requires the evolution time $T$ to be larger than the relaxation time $T_{\gamma} \sim 1 / \gamma$. If this condition is satisfied then the stationary action (19) is related to the spectral density as

$$
\tilde{I}=\int_{-\infty}^{\infty} P(v) d v
$$

Notice that, unlike the stationary action $\tilde{I}$, the spectral density $P(v)$ does depend on the oscillator nonlinearity. Different curves in the inset in Fig. 1 show the spectral density of the oscillator (14) for $\omega=1$ and increasing value of $g$. It is seen that with increase of $g$ the maximum of the spectral density shifts to the higher (negative) frequencies. A qualitative explanation for this shift is that for nonzero nonlinearity the oscillator eigenfrequency $\Omega=\omega+g I$ depends on the action $I$ which in the stationary regime is distributed according to the exponential law $f(I)=\sigma^{-2} \exp \left(-I / \sigma^{2}\right), \sigma^{2}=D / \gamma$. On the quantitative level, calculation of the spectral density requires sophisticated methods which involve continued fraction expansion [28]. The only exclusion is the case $g=0$, where $P(v)$ can be calculated exactly by using the formalism of the Green function [29], which gives

$$
P(\nu)=\frac{D}{2 \pi} \frac{1}{(\nu+\omega)^{2}+(\gamma / 2)^{2}} .
$$

\section{CHAIN OF COUPLED OSCILLATORS}

Now we are prepared to address the current in the $\mathrm{BH}$ chain of length $L$ connecting two reservoirs. Generalizing the results of the previous section for system (2), the equation for the distribution function $f=f\left(\mathbf{a}, \mathbf{a}^{*} ; t\right), \mathbf{a}=\left(a_{1}, \ldots, a_{L}\right)$, reads

$$
\frac{\partial f}{\partial t}=\{H, f\}+\sum_{l=1, L} \mathcal{D}^{(l)}(f)+\sum_{l=1, L} \mathcal{G}^{(l)}(f),
$$

where

$$
\mathcal{D}^{(l)}(f)=D_{l} \frac{\partial^{2} f}{\partial a_{l} \partial a_{l}^{*}}
$$

and

$$
\mathcal{G}^{(l)}(f)=\frac{\gamma_{l}}{2}\left(a_{l} \frac{\partial f}{\partial a_{l}}+2 f+a_{l}^{*} \frac{\partial f}{\partial a_{l}^{*}}\right) .
$$

Thus, in the pseudoclassical approach the original quantum problem corresponds to the system of $L$ coupled nonlinear oscillators,

$$
\begin{aligned}
H & =\sum_{l=1}^{L} H_{l}-\frac{J}{2}\left(\sum_{l} a_{l+1}^{*} a_{l}+\text { c.c. }\right), \\
H_{l} & =\omega I_{l}+\frac{g}{2} I_{l}^{2}, \quad I_{l}=\left|a_{l}\right|^{2},
\end{aligned}
$$

where the first and the last oscillators have nonzero friction coefficients $\gamma_{1}$ and $\gamma_{L}$ and are driven by stochastic forces with amplitudes $\sqrt{D_{1} / 2}$ and $\sqrt{D_{L} / 2}$, respectively:

$$
i \dot{a}_{l}=\frac{\partial H}{\partial a_{l}^{*}}-i \frac{\gamma_{l}}{2}\left(\delta_{1, l}+\delta_{L, l}\right) a_{l}+\sqrt{\frac{D_{l}}{2}}\left(\delta_{1, l}+\delta_{L, l}\right) \xi_{l}(t) .
$$

Two remarks are in turn. First, there is some freedom in defining the effective Planck constant $\hbar^{\prime}$ for the considered system. In what follows we use $\hbar^{\prime}=1 / \bar{n}_{1}$ where, as before, $\bar{n}_{1}$ is the particle density of the left reservoir. Then the macroscopic interaction constant $g$ in Eq. (27) is related to the microscopic interaction constant $U$ in Eq. (2) as $g=$ $U \bar{n}_{1}$. Notice that, since we assume $\bar{n}_{1}>\bar{n}_{L}$, the above choice ensures $I_{l} \leqslant 1$ for every oscillator.

The second comment concerns the lower boundary for $\bar{n}_{1}$ where the pseudoclassical approach is still a good approximation. This is largely an open question because answering it requires knowledge of the quantum dynamics. Unfortunately, numerical solution of master equation (1) is feasible only for a few-site system. In Fig. 2 we compare the exact and pseudoclassical results for $L=3$. (The quantities depicted in the figure are discussed in the next section). A reasonable agreement between the dash-dotted and solid lines indicates that for this system size one can go down to $\bar{n}_{1}=2$. Although the discussed lower boundary is not crucial for the results reported below, it should be remembered that this boundary does 


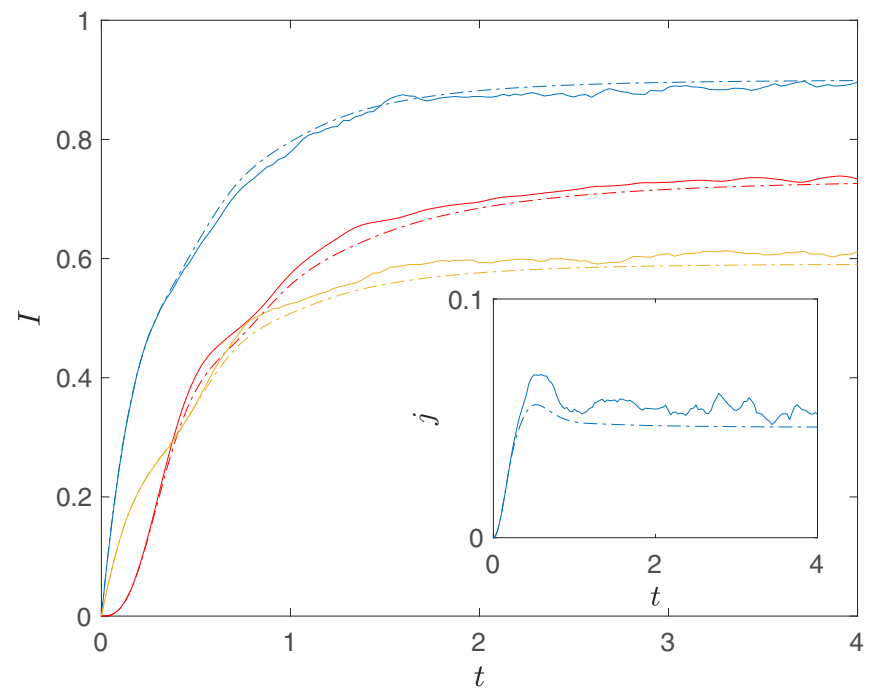

FIG. 2. Comparison between the exact (dash-dotted lines) and pseudoclassical (solid lines) results for $L=3, J=1, \bar{n}_{1}=2, \bar{n}_{L}=$ $1, \gamma=0.5\left(D_{1}=\gamma, D_{L}=\gamma \bar{n}_{L} / \bar{n}_{1}\right)$, and $U=0.5(g=1)$. In the quantum case the Fock basis is truncated to $\sum_{l} n_{l} \leqslant 20$, which gives the total dimension of the Hilbert space, $\mathcal{N}=1771$. In the pseudoclassical case the average is taken over 16000 realizations of the stochastic processes.

exist. We plan to quantify the error due to the pseudoclassical approximation in a separate publication.

\section{A. Single-particle density matrix}

To calculate the current of the bosonic carriers in the open BH chain it suffices to know the single-particle density matrix (SPDM) whose elements are defined as

$$
\rho_{l, m}(t)=\operatorname{Tr}\left[\hat{a}_{l}^{\dagger} \hat{a}_{m} \mathcal{R}(t)\right], \quad 1 \leqslant l, m \leqslant L .
$$

In the pseudoclassical approach Eq. (29) obviously takes the form

$$
\rho_{l, m}(t)=\int a_{l}^{*} a_{m} f\left(\mathbf{a}, \mathbf{a}^{*} ; t\right) d \mathbf{a} d \mathbf{a}^{*}
$$

if we use the formalism of the Fokker-Planck equation (24), or

$$
\rho_{l, m}(t)=\overline{a_{l}^{*}(t) a_{m}(t)}
$$

if we use the Langevin equation (28). Knowing the SPDM, the current density $j(t)$ is then found as

$$
j(t)=\frac{J}{L-1} \sum_{l=1}^{L-1} \operatorname{Im}\left[\rho_{l, l+1}(t)\right] .
$$

If $g=0$, matrix (30) can be found analytically. Taking the time derivative from both sides of Eq. (30) and substituting there Eq. (24) we obtain after integrating by parts the following system of equations on the matrix elements:

$$
\begin{aligned}
\frac{d}{d t} \rho_{l, m}= & i \frac{J}{2}\left(\rho_{l, m+1}+\rho_{l, m-1}-\rho_{l+1, m}-\rho_{l-1, m}\right) \\
& -\sum_{j=1, L} \frac{\gamma_{j}}{2}\left(\delta_{l, j}+\delta_{m, j}\right) \rho_{l, m}+\sum_{j=1, L} D_{j} \delta_{l, j} \delta_{m, j} .
\end{aligned}
$$
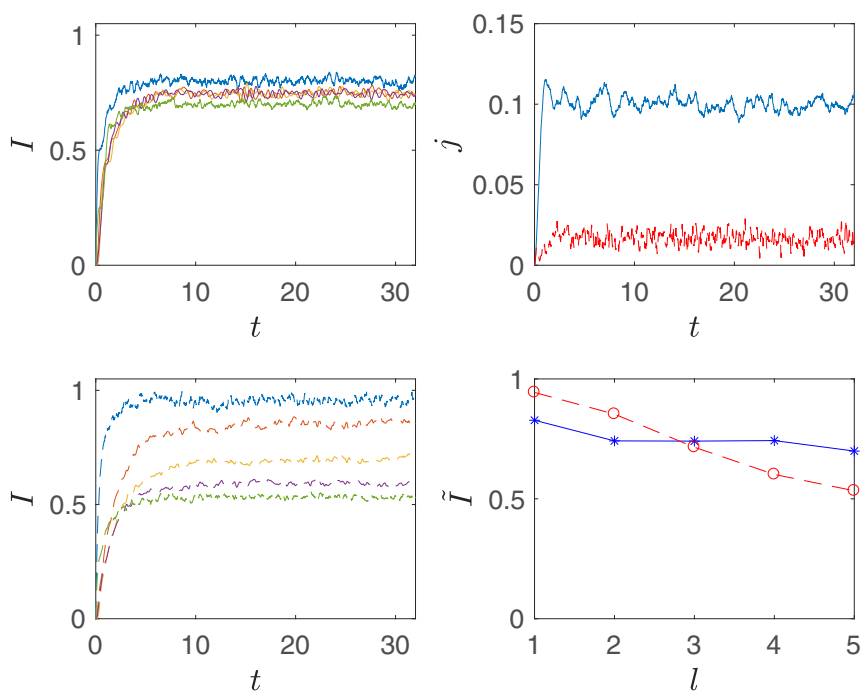

FIG. 3. Comparison between the cases $g=0$ and $g=2$ where the latter case is indicated by dashed lines. Left column, dynamics of the mean actions $I_{l}(t)$; upper right, dynamics of the mean current; and lower right, stationary values of the actions $\tilde{I}_{l}$ along the chain. The other parameters are $\gamma_{1}=\gamma_{L}=0.5, D_{1}=0.5$, and $D_{L}=0.25$. Average over 4000 runs.

This equation coincides with that obtained quantum mechanically in Refs. [12,13]. Thus, in the case of vanishing interparticle interactions the pseudoclassical approach is exact. The stationary solution of Eq. (33) is the three diagonal matrix with pure imaginary off-diagonal elements which determine the stationary current $\tilde{j}$. In what follows we consider the case $\gamma_{1}=\gamma_{L} \equiv \gamma$ where the analytical expression for the stationary current is particularly simple:

$$
\tilde{j}=j_{0} \frac{J \gamma}{J^{2}+\gamma^{2}} \frac{D_{1}-D_{L}}{2 \gamma} .
$$

Notice that the current is proportional to the difference of diffusion coefficients. (In the quantum problem, it is proportional to the difference $\left.\bar{n}_{1}-\bar{n}_{L}\right)$. On the contrary, the stationary actions $\tilde{I}_{l}$ (i.e., the diagonal elements of the SPDM) are proportional to the sum of diffusion coefficients,

$$
\tilde{I}_{l}=\frac{D_{1}+D_{L}}{2 \gamma}
$$

Exclusions are the first (last) oscillator whose stationary actions are slightly above (below) the value indicated in Eq. (35).

\section{B. Nonlinear versus linear chain}

We proceed with the case of interacting particles. To test our numerical code we first run it for $g=0$. The upper-left panel in Fig. 3 shows dynamics of the diagonal elements $\rho_{l, l}(t)$ of the SPDM for $L=5, \gamma_{1}=\gamma_{L}=0.5, D_{1}=0.5$, $D_{L}=0.25$, and the initial conditions $\rho_{l, l}(t=0)=0$; i.e., initially all oscillators are at rest. It is seen that oscillators rapidly approach the steady state with the stationary actions $\tilde{I}_{l}$ given by Eq. (35) (see the solid line in the lower-right panel). The dynamics of the mean current $j(t)$ is depicted in the upper-right panel by the solid blue line. Again, it is seen that the current approaches the value given by Eq. (34). The 

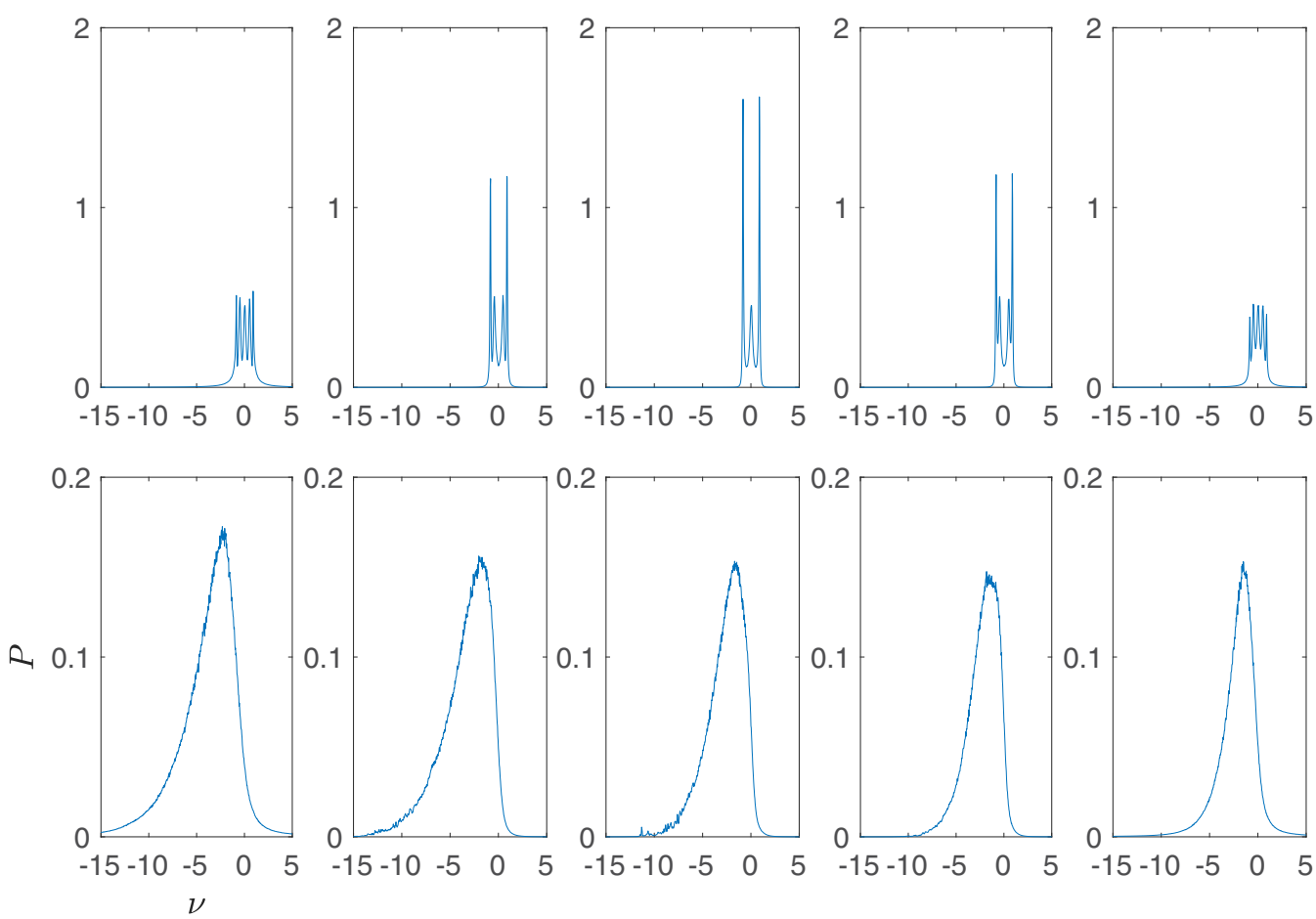

FIG. 4. Oscillator spectral density for $g=0$ (top) and $g=2$ (bottom). The other parameters are the same as in Fig. 3.

dashed lines in Fig. 3 depict the case of interacting particles. A drastic decrease of the stationary current is noticed.

One finds a qualitative explanation for the observed effects by considering the oscillator spectral densities. Panels in the upper row in Fig. 4 show the spectral densities of oscillators in the chain for $g=0$. It is easy to identify in this figure frequencies $\omega_{k}$ of the collective modes which are given by the real part of complex poles of the Green function for the system of linear differential equations (28) with omitted stochastic terms. For moderate values of the decay constants they can be approximated by eigenfrequencies of the linear chain,

$$
\omega_{k}=-J \cos (2 \pi k / L), \quad l=1, \ldots, L,
$$

which are located in the "conductance" band $\left|\omega_{k}\right| \leqslant J$. Thus, the driving force acting on the first and last oscillator excites the collective modes. This leads to an efficient transport of excitations from the first to the last oscillator that quantummechanically corresponds to the flow of bosonic particles from the left to the right reservoir. Since the current is independent of the chain length $L$ we refer to this dynamical regime as the ballistic transport.

The lower panels in Fig. 4 show the spectral densities of oscillators for $g=2$. Now the system has no collective modes and transmission of excitations is a diffusionlike process [compare $P(v)$ in lower panels with $P(v)$ in the inset in Fig. 1]. As a consequence, we observe considerable decrease in the stationary current. Furthermore, contrary to the ballistic regime, in the diffusive regime the current depends on the chain length $L$. We discuss this dependence in the next section.

\section{The limit $L \rightarrow \infty$}

We performed extensive numerical simulations of the system dynamics for a large system size up to $L=100$. The open circles in the right-hand panel in Fig. 5 show the stationary current $\tilde{j}$ as a function of $1 / L$. A linear dependence is noticed that is consistent with the above conjecture about the diffusive transport. We mention that the change of the transport regime from ballistic to diffusive with increase of $g$ is a crossover rather than a phase transition. The necessary condition for this crossover is that the nonlinear shift of the oscillator frequencies, which is estimated as $g \tilde{I}_{l}$, exceeds the width of the conductance band, i.e.,

$$
\tilde{I}_{l}>J / g
$$

We stress that condition (37) has to be satisfied for all oscillators to get the universal dependence $\tilde{j} \sim 1 / L$. This, for example, is not the case if we set $D_{L}$ to zero. (In the original
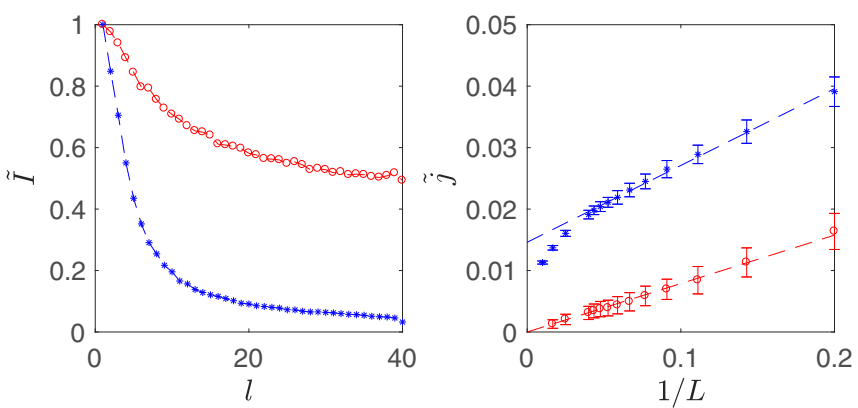

FIG. 5. Left: The mean oscillator actions along the chain of length $L=40$ for $D_{L}=D_{1} / 2$ (open circles) and $D_{L}=0$ (asterisks). The other parameters are $g=2, D_{1}=0.5$, and $\gamma_{1}=\gamma_{L}=0.5$. Average over 4000 realizations. Right: The stationary current as a function of the inverse chain length for two considered cases $D_{L}=$ 0.25 (open circles) and $D_{L}=0$ (asterisks). The bars show statistical error due to the finite number of realizations. 
quantum problem this corresponds to pure sink of particle at the right edge of the chain.) Here condition (37) is satisfied for oscillators in the left part of the chain but violated for oscillators in the right part (see the left-hand panel in Fig. 5). As a consequence, the spectral densities of oscillators in the left and right parts of the chain resemble those for the diffusive and ballistic transport, respectively. The dependence of the stationary current on the system size for this mixed regime is depicted by asterisks in the right-hand panel in Fig. 5. It is seen that the presence of a "ballistic part" results in a larger current as compared to the pure diffusive transport regime. The depicted numerical data also indicate a highly nontrivial dependence of the stationary current on the difference $\bar{n}_{1}-\bar{n}_{L}$ which involves the chain length $L$ as an additional parameter.

\section{CONCLUSION}

We analyze stationary current of the bosonic carriers in the Bose-Hubbard chain of length $L$ where the first and the last sites of the chain are attached to reservoirs of Bose particles. The analysis is carried out by using the pseudoclassical approach which reduces the original quantum problem to the classical problem for $L$ coupled oscillators where the first and last oscillators are subject to both friction and excitation. In the case of vanishing interparticle interactions we analytically calculate elements $\rho_{l, m}$ of the single-particle density matrix which classically correspond to the correlation functions between the $l$ th and $m$ th oscillators. It is shown that the pseudoclassical approach reproduces the quantummechanical results exactly. In particular, the stationary current (which is determined by off-diagonal elements of the stationary single-particle density matrix) is proportional to the particle density difference $\Delta \bar{n}=\bar{n}_{1}-\bar{n}_{L}$ of the reservoirs and is independent of the chain length $L$.

Next we numerically address the case of finite interparticle interactions which make the classical oscillators nonlinear. A drastic reduction of the stationary current as compared to the linear case is noticed. Furthermore, the current depends on the chain length and in the case of small difference $\Delta \bar{n} \ll \bar{n}_{1}$ scales as $1 / L$. We give a qualitative explanation for this change in the transport regime by introducing and analyzing the spectral densities of oscillators. We stress that the oscillator spectral density explicitly involves the notion of trajectory and, hence, is meaningful only in the classical approach. Nevertheless, we find this quantity quite helpful for understanding different quantum transport regimes in the open Bose-Hubbard chain.

\section{ACKNOWLEDGMENT}

This work has been supported through Russian Science Foundation (RU), N19-12-00167.
[1] R. Gati and M. K. Oberthaler, A bosonic Josephson junction, J. Phys. B 40, R61 (2007).

[2] J. Esteve, C. Gross, A. Weller, S. Giovanazzi, and M. K. Oberthaler, Squeezing and entanglement in a Bose-Einstein condensate, Nature (London) 455, 1216 (2008).

[3] F. Meinert, M. J. Mark, E. Kirilov, K. Lauber, P. Weinmann, M. Gröbner, and H.-C. Nägerl, Interaction-Induced Quantum Phase Revivals and Evidence for the Transition to the Quantum Chaotic Regime in 1D Atomic Bloch Oscillations, Phys. Rev. Lett. 112, 193003 (2014).

[4] C. J. Fujiwara, K. Singh, Z. A. Geiger, R. Senaratne, S. V. Rajagopal, M. Lipatov, and D. M. Weld, Transport in FloquetBloch Bands, Phys. Rev. Lett. 122, 010402 (2019).

[5] R. Labouvie, B. Santra, S. Heun, S. Wimberger, and H. Ott, Negative Differential Conductivity in an Interacting Quantum Gas, Phys. Rev. Lett. 115, 050601 (2015).

[6] R. Labouvie, B. Santra, S. Heun, and H. Ott, Bistability in a Driven-Dissipative Superfluid, Phys. Rev. Lett. 116, 235302 (2016).

[7] J. Brantut, J. Meineke, D. Stadler, S. Krinner, and T. Esslinger, Conduction of ultracold fermions through a mesoscopic channel, Science 337, 1069 (2012).

[8] S. Krinner, D. Stadler, D. Husmann, J. P. Brantut, and T. Esslinger, Observation of quantized conductance in neutral matter, Nature (London) 517, 64 (2015).

[9] M. Lebrat, P. Grisins, D. Husmann, S. Häusler, L. Corman, T. Giamarchi, J.-Ph. Brantut, and T. Esslinger, Band and Correlated Insulators of Cold Fermions in a Mesoscopic Lattice, Phys. Rev. X 8, 011053 (2018).
[10] A. Ivanov, G. Kordas, A. Komnik, and S. Wimberger, Bosonic transport through a chain of quantum dots, Eur. Phys. J. B 86, 345 (2013).

[11] G. Kordas, D. Witthaut, P. Buonsante, A. Vezzani, R. Burioni, A. I. Karanikas, and S. Wimberger, The dissipative Bose-Hubbard model, Eur. Phys. J. Spec. Top. 224, 2127 (2015).

[12] G. Kordas, D. Witthaut, and S. Wimberger, Non-equilibrium dynamics in dissipative Bose-Hubbard chains, Ann. Phys. (Berlin) 527, 619 (2015).

[13] A. R. Kolovsky, Z. Denis, and S. Wimberger, LandauerBüttiker equation for bosonic carriers, Phys. Rev. A 98, 043623 (2018).

[14] T. Prosen and B. Žunkovič, Exact solution of Markovian master equations for quadratic Fermi systems: Thermal baths, open XY spin chains and non-equilibrium phase transition, New J. Phys. 12, 025016 (2010).

[15] M. Znidaric, Exact solution for a diffusive nonequilibrium steady state of an open quantum chain, J. Stat. Mech. (2010) L05002.

[16] T. Prosen, Exact Nonequilibrium Steady State of an Open Hubbard Chain, Phys. Rev. Lett. 112, 030603 (2014).

[17] B. Buca and T. Prosen, Charge and spin current statistics of the open Hubbard model with weak coupling to the environment, Phys. Rev. E 95, 052141 (2017).

[18] K. W. Mahmud, H. Perry, and W. P. Reinhardt, Quantum phasespace picture of Bose-Einstein condensates in a double well, Phys. Rev. A 71, 023615 (2005). 
[19] S. Mossmann and C. Jung, Semiclassical approach to BoseEinstein condensates in a triple well potential, Phys. Rev. A 74, 033601 (2006).

[20] E. M. Graefe and H. J. Korsch, Semiclassical quantization of an $N$-particle Bose-Hubbard model, Phys. Rev. A 76, 032116 (2007).

[21] F. Trimborn, D. Witthaut, and H. J. Korsch, Exact number conserving phase-space dynamics of the $M$-site Bose-Hubbard model, Phys. Rev. A 77, 043631 (2008).

[22] A. R. Kolovsky, H.-J. Korsch, and E.-M. Graefe, Bloch oscillations of Bose-Einstein condensates: Quantum counterpart of dynamical instability, Phys. Rev. A 80, 023617 (2009).

[23] T. Zibold, E. Nicklas, C. Gross, and M. K. Oberthaler, Classical Bifurcation at the Transition from Rabi to Josephson Dynamics, Phys. Rev. Lett. 105, 204101 (2010).
[24] A. R. Kolovsky, Treating many-body quantum systems by means of classical mechanics, Springer Proc. Phys. 191, 37 (2017).

[25] A. A. Bychek, D. N. Maksimov, and A. R. Kolovsky, NOON state of Bose atoms in the double-well potential via an excitedstate quantum phase transition, Phys. Rev. A 97, 063624 (2018).

[26] A. A. Bychek, P. S. Muraev, and A. R. Kolovsky, Probing quantum chaos in many-body quantum systems by the induced dissipation, Phys. Rev. A 100, 013610 (2019).

[27] K. Vogel and H. Risken, Quantum-tunneling rates and stationary solutions in dispersive optical bistability, Phys. Rev. A 42, 627 (1990).

[28] M. Bixon and R. Zwanzig, Brownian motion of a nonlinear oscillator, J. Stat. Phys. 3, 245 (1971).

[29] S. A. Akhmanov, Yu. E. Diyakov, and A. S. Chirkin, Introduction to Statistical Radiophysics and Optics (Nauka, Moscow, 1981), in Russian. 